$\mathrm{DTP} / 99 / 100$

November 1999

\title{
Unintegrated parton distributions and prompt photon hadroproduction
}

\author{
M.A. Kimber ${ }^{a}$, A.D. Martin $^{a}$ and M.G. Ryskin ${ }^{a, b}$ \\ ${ }^{a}$ Department of Physics, University of Durham, Durham, DH1 3LE \\ ${ }^{b}$ Petersburg Nuclear Physics Institute, Gatchina, St. Petersburg, 188350, Russia
}

\begin{abstract}
We introduce a general expression which enables the parton distribution, unintegrated over the parton transverse momentum, to be obtained from the conventional parton densities. We use the formalism to study the effects of the transverse momentum $q_{t}$ of the incoming partonic system on the calculation of the transverse momentum spectra of prompt photons produced in high energy $p p$ and $p \bar{p}$ collisions. For the purposes of illustration, we use the double logarithm approximation. For large $q_{t}$ we calculate the effect directly from the perturbative formalism, whereas for small $q_{t}$ we bound the effect using two extreme hypotheses. In both $q_{t}$ domains we find that the shapes of the prompt photon spectra are not significantly modified, although the cross sections are enhanced.
\end{abstract}

\section{Introduction}

The cross sections for hard hadronic processes are conventionally described in terms of universal parton distributions $a\left(x, \mu^{2}\right)$, with $a=x q$ or $x g$, convoluted with the cross sections of the partonic subprocesses calculated in perturbative QCD at some large scale $\mu$ characteristic of the subprocess. For example, $\mu$ may be the transverse momentum of an outgoing parton or the mass of a heavy quark should it participate in the subprocess. In this paper we present the formalism which enables the parton distributions $f_{a}\left(x, k_{t}^{2}, \mu^{2}\right)$, unintegrated over the parton transverse momentum $k_{t}$, to be constructed from the conventional integrated distributions 
$a\left(x, \lambda^{2}\right)$. We will see that it is important to distinguish the scale $\lambda$ from the hard scale $\mu$. We first give the general prescription, and then we take the limits which correspond to the leading $\log (1 / x)$ BFKL approach [1] and to the double log DDT prescription [2].

These unintegrated parton densities $f_{a}\left(x, k_{t}^{2}, \mu^{2}\right)$ should be used to describe any hard hadronic process, such as heavy quark production or the production of large $E_{T}$ jets [3]. The only exception is deep inelastic scattering (DIS). If the $k_{t}$ integration is performed in the convolution of the unintegrated parton distributions with the deep inelastic partonic subprocess then the remaining $x$ convolution is in terms of just the conventional parton distributions'.

To demonstrate the use of the formalism we study a particularly relevant and topical process, namely prompt photon hadroproduction. The production of prompt photons in high energy $p p$ collisions has long played a key role in constraining the gluon distribution of the proton at large $x$ through the dominance of the subprocess $g q \rightarrow \gamma q$. The reason is that the gluon enters at leading order, unlike its contribution to the description of deep inelastic scattering. However the description of the transverse momentum spectrum of the produced photons is more problematic than inclusive deep inelastic scattering. The observed $p_{t \gamma}$ spectrum in $p p \rightarrow \gamma X$ (or $p \bar{p} \rightarrow \gamma X$ ) is found to be steeper than the prediction of perturbative QCD [4, 5]. The explanation of this discrepancy is usually attributed to the intrinsic transverse momenta $k_{t}$ of the incoming partons, which are usually assumed to have a Gaussian-like $k_{t}$ distribution [6, 7]. Thus part of the observed $p_{t \gamma}$ comes from the initial partonic $k_{t}$ such that the hard subprocess singularity $d \hat{\sigma} / d \hat{t} \sim 1 / p_{t \gamma}^{4}$ is approached more closely, and hence leads to a steeper $p_{t \gamma}$ spectrum. However in order to describe the observed spectra it is necessary to introduce a $k_{t}$ spectrum with an average value which increases from $\left\langle k_{t}\right\rangle \sim 0.5 \mathrm{GeV}$ to more than $2 \mathrm{GeV}$ [6] as the collision energy $\sqrt{s}$ increases from UA6, E706 [8, 9] to Tevatron [10] energies. Such large partonic $\left\langle k_{t}\right\rangle$ cannot originate solely from the large distance confinement domain, but must also have a significant perturbative QCD component. Indeed it is easy to show that perturbative QCDE gives a non-zero $k_{t}$ with a distribution in which $\left\langle k_{t}\right\rangle$ increases as $\sqrt{s}$. Ref. [12] also contains a discussion of the partonic $k_{t}$ generated by perturbative QCD, but comparison with the $p_{t \gamma}$ data is based on a phenomenological Gaussian $k_{t}$ distribution.

Here we study the role of both the non-perturbative and perturbative components of the incoming partonic $k_{t}$ in describing the observed $p_{t \gamma}$ spectrum in high energy prompt photon hadroproduction, that is $p p$ (or $p \bar{p}) \rightarrow \gamma X$. Perturbative QCD is applied, at leading order, in the domain of large partonic transverse momentum $k_{t}>q_{0}$, where $q_{0} \sim 1-2 \mathrm{GeV}$ is the starting point of DGLAP evolution in the global parton analyses [0, 13]. In the small $k_{t}<q_{0}$ region it proves sufficient to consider two extreme hypotheses for the normalised non-perturbative or intrinsic $k_{t}$ spectrum:

\footnotetext{
${ }^{1} \mathrm{~A}$ somewhat analogous example is the DIS renormalization scheme, where the higher order corrections are present in any process except DIS, which is used as the reference process.

${ }^{2}$ Another correction to the perturbative form of the $p_{t \gamma}$ spectrum comes from the resummation of $\log \left(1-x_{T}\right)$ terms, where $x_{T} \equiv 2 p_{t \gamma} / \sqrt{s}$ 11. This effect only changes the spectrum at the larger values of $x_{T}$, close to the kinematic boundary.
} 
(a) a $\delta\left(k_{t}^{2}\right)$ distribution, i.e. no intrinsic $k_{t}$ from the confinement region,

(b) a wide Gaussian distribution of width $\left\langle k_{t}^{2}\right\rangle \sim\left\langle q_{0}^{2}\right\rangle$.

From these extreme possibilities we conclude that the intrinsic partonic transverse momentum has a rather small effect on the $p_{t \gamma}$ spectrum. Moreover we find the effects of $k_{t}$ from the perturbative domain $\left(k_{t}>q_{0}\right)$ do not significantly change the $p_{t \gamma}$ spectrum.

In Section 2 we introduce the unintegrated parton distributions and the parton-parton luminosity function $\mathcal{L}_{a b}\left(x_{1}, x_{2}, q_{t}\right)$, which is the probability of finding the incoming $a, b$ partons (with momenta specified by $x_{1}, k_{1 t}^{2}$ and $x_{2}, k_{2 t}^{2}$ ) with net transverse momentum $\boldsymbol{q}_{t}=\boldsymbol{k}_{t 1}+\boldsymbol{k}_{t 2}$. In Section 3 we apply the formalism to the production of prompt photons in high energy $p p$ or $p \bar{p}$ collisions. We convolute the luminosity functions with the hard $g q \rightarrow \gamma q$ and $q \bar{q} \rightarrow \gamma g$ subprocess cross sections, taking into account the boost of the final $\gamma+$ parton system coming from the incoming partonic $q_{t}$. In Section 4 , we show the effect of both the perturbative and non-perturbative components of $q_{t}$ on the predictions for the $p_{t \gamma}$ distributions at different $\sqrt{s}$, together with the measured $p_{t \gamma}$ spectra. Finally in Section 5 we present our conclusions.

\section{Unintegrated partons and the luminosity function}

To explore the effects of partonic transverse momenta we need to know the distribution of a parton $a$, say as a function of its transverse momentum $k_{t}$, as well as of $x$. A straightforward way to obtain such a distribution is to consider the DGLAP evolution?

$$
\frac{\partial a}{\partial \ln \lambda^{2}}=\frac{\alpha_{S}}{2 \pi}\left[\int_{x}^{1-\Delta} P_{a a^{\prime}}(z) a^{\prime}\left(\frac{x}{z}, \lambda^{2}\right) d z-a\left(x, \lambda^{2}\right) \sum_{a^{\prime}} \int_{0}^{1-\Delta} P_{a^{\prime} a}\left(z^{\prime}\right) d z^{\prime}\right],
$$

where the (integrated) parton density, $a\left(x, \lambda^{2}\right)$, denotes $x g\left(x, \lambda^{2}\right)$ or $x q\left(x, \lambda^{2}\right)$. The first term on the right-hand-side describes the number of partons $\delta a$ emitted in the interval $\lambda^{2}<k_{t}^{2}<$ $\lambda^{2}+\delta \lambda^{2}$, while the second (virtual) term reflects the fact that the parton $a$ disappears after the splitting. The second contribution may be resummed to give the survival probability $T_{a}$ that the parton $a$ with transverse momentum $k_{t}$ remains untouched in the evolution up to the factorization scale. The survival probability is given by the double logarithmic Sudakov factor 14

$$
T_{a}\left(k_{t}, \mu\right)=\exp \left(-\int_{k_{t}^{2}}^{\mu^{2}} \frac{\alpha_{S}\left(p_{t}\right)}{2 \pi} \frac{d p_{t}^{2}}{p_{t}^{2}} \sum_{a^{\prime}} \int_{0}^{1-\Delta} P_{a^{\prime} a}\left(z^{\prime}\right) d z^{\prime}\right) .
$$

Thus the probability to find a parton $a$ with transverse momentum $k_{t}$ which initiates our hard process, with factorization scale $\mu$, is

$$
f_{a}\left(x, k_{t}^{2}, \mu^{2}\right)=\left(\frac{\alpha_{S}\left(k_{t}\right)}{2 \pi} \int_{x}^{1-\Delta} P_{a a^{\prime}}(z) a^{\prime}\left(\frac{x}{z}, k_{t}^{2}\right) d z\right) T_{a}\left(k_{t}, \mu\right) .
$$

\footnotetext{
${ }^{3}$ For the $g \rightarrow g g$ splitting we need to insert a factor $z^{\prime}$ in the last integral of (㑑) to account for the identity of the produced gluons.
} 
Now we have to specify the value of the infrared cut-off $\Delta$, which is introduced to protect the $1 /\left(1-z^{\prime}\right)$ singularity in the splitting functions arising from soft gluon emission. In the original DGLAP equation (11) for integrated partons this singularity is cancelled between the real and virtual contributions. However after the resummation of the virtual terms the real soft gluon emission has to be accounted for explicitly as it changes the transverse momentum of the parton. That is we have to find the physically appropriate choice of the cut-off $\Delta$, which comes from the coherence effect [14]. The most convenient way is to go to the Breit frame where the angular ordering condition becomes the requirement that no soft gluons are emitted in the backward direction. In this frame the parton energy $E=\mu$. Then the integral over the soft gluon momentum $d z^{\prime} /\left(1-z^{\prime}\right)=d \omega / \omega$ covers the interval $k_{t}<\omega<E$, where $\omega$ is the energy of the soft gluon. That is we take $\Delta=k_{t} / E=k_{t} / \mu$. Of course the same $\Delta$ must be used in both the real emission integral in (3) and in the survival probability $T$ in (2). Below we consider two limits of the general expression (3) for the unintegrated parton density.

In the leading $\log (1 / x)$ or BFKL limit the virtual contribution in the DGLAP equation, (1), is neglected and the survival probability $T=1$. Hence we come back to the familiar prescription

$$
f_{a}\left(x, k_{t}^{2}\right)=\left.\frac{\partial\left(a\left(x, \lambda^{2}\right)\right)}{\partial \ln \lambda^{2}}\right|_{\lambda^{2}=k_{t}^{2}} .
$$

Note that in this limit the unintegrated parton density does not depend on $\mu$, since there are no $\log (1 / x)$ terms in the scale dependent part.

In the double logarithm limit, (3) may be written

$$
f_{a}\left(x, k_{t}^{2}, \mu^{2}\right)=\left.\frac{\partial}{\partial \ln \lambda^{2}}\left[a\left(x, \lambda^{2}\right) T_{a}(\lambda, \mu)\right]\right|_{\lambda=k_{t}}
$$

with survival probability

$$
T_{a}(\lambda, \mu)=\exp \left(-\int_{\lambda^{2}}^{\mu^{2}} \frac{\alpha_{S}\left(p_{t}\right)}{2 \pi} \frac{d p_{t}^{2}}{p_{t}^{2}} \int_{0}^{1-\Delta} \frac{d z^{\prime}}{1-z^{\prime}} 2 C_{a}\right)
$$

where $C_{q}=C_{F}=\left(N_{C}^{2}-1\right) / 2 N_{C}$ and $C_{g}=C_{A}=N_{C}$. In this limit the cut-off $\Delta$ is numerically small so one can choose the value of $\Delta$ in (西) equal to the value of $\Delta$ in (6). Therefore the second term of the right-hand-side of (11) cancels the derivative $\partial T_{a} / \partial \ln \lambda^{2}$. To be precise, the only double logarithmic contribution comes from the singular $1 /\left(1-z^{\prime}\right)$ part of the diagonal splitting function $P_{a a}\left(z^{\prime}\right)$ in (1) which exactly equals the last integrand $2 C_{a} /\left(1-z^{\prime}\right)$ in (6).

To obtain the parton-parton luminosity function we have to perform a convolution over the distributions of the two incoming partons $a, b$

$$
\mathcal{L}_{a b}\left(x_{1}, x_{2}, q_{t}\right)=\int f_{a}\left(x_{1}, k_{1}^{2}, \mu^{2}\right) f_{b}\left(x_{2}, k_{2}^{2}, \mu^{2}\right) \delta^{(2)}\left(\boldsymbol{k}_{1}+\boldsymbol{k}_{2}-\boldsymbol{q}_{t}\right) \frac{d^{2} k_{1} d^{2} k_{2}}{\pi k_{1}^{2} k_{2}^{2}},
$$

where, for simplicity, we have omitted the $t$ subscript on the $k_{i}$. The luminosity element, $\mathcal{L}_{a b} d q_{t}^{2}$, gives the probability that the incoming partons have a net square transverse momentum in the 
interval $\left(q_{t}^{2}, q_{t}^{2}+d q_{t}^{2}\right)$. To leading order all the transverse momenta are strongly ordered, and so either $k_{1} \ll k_{2} \simeq q_{t}$ or $k_{2} \ll k_{1} \simeq q_{t}$. In the first case we may integrate over $k_{1}$ giving

$$
\int^{q_{t}} f_{a} f_{b} \frac{d^{2} k_{1}}{\pi k_{1}^{2}}=\left[a\left(x_{1}, q_{t}^{2}\right) T_{a}\left(q_{t}, \mu\right)\right] f_{b}\left(x_{2}, q_{t}^{2}, \mu^{2}\right),
$$

and vice versa for $k_{2} \ll k_{1}$. Thus the sum of both contributions may be expressed in the compact form

$$
\mathcal{L}_{a b}\left(x_{1}, x_{2}, q_{t}\right)=\left.\frac{\partial}{\partial \lambda^{2}}\left[a\left(x_{1}, \lambda^{2}\right) T_{a}(\lambda, \mu) b\left(x_{2}, \lambda^{2}\right) T_{b}(\lambda, \mu)\right]\right|_{\lambda=q_{t}} .
$$

A similar expression for the Drell-Yan process was originally obtained in the classic work of ref. [2].

\section{The prompt photon $p_{t \gamma}$ distribution}

To obtain the cross section for inclusive prompt photon hadroproduction we convolute the hard subprocess cross sections for $g q \rightarrow \gamma q$ etc. with the parton luminosity of (9). We obtain

$$
\begin{aligned}
E_{\gamma} \frac{d \sigma}{d^{3} p_{\gamma}}= & \frac{d \sigma}{d \eta_{\gamma} \pi d p_{t \gamma}^{2}}=\int \mathcal{L}\left(x_{1}, x_{2}, q_{t}\right) \frac{d x_{1}}{x_{1}} \frac{d x_{2}}{x_{2}} d q_{t}^{2} \\
& \times \frac{d \hat{\sigma}}{d \hat{t}} d \hat{t} \frac{d \phi}{2 \pi} \delta\left(\eta_{\gamma}-\ldots\right) \delta\left(p_{t \gamma}^{2}-\ldots\right) \theta\left(\mu^{2}-q_{t}^{2}\right) \theta\left(|\hat{t}|-q_{t}^{2}\right)
\end{aligned}
$$

where $\phi$ is the azimuthal angle between $\boldsymbol{q}_{t}$ and the photon transverse momentum $\boldsymbol{p}_{t}^{\prime}$ in the hard subprocess. That is $p_{t}^{\prime}=\frac{1}{2} M \sin \theta$, where $\theta$ is the polar scattering angle in the hard subprocess and $M^{2}=\hat{s}$ is the invariant mass squared of the produced $\gamma q$ system. We thus have

$$
\hat{t}=-\frac{M^{2}}{2}(1-\cos \theta)
$$

in the hard subprocess cross section $d \hat{\sigma} / d \hat{t}$. The theta functions in (10) impose the physical requirements that the partonic transverse momentum should be less than, first, the scale $\mu$ and, second, the momenta in the hard subprocess. The last condition comes from the fact that at leading order all the transverse momenta along the chain are strongly ordered. For the hard subprocess initiated by the gluon $\left(k_{1}\right)$ and the quark $\left(k_{2}\right)$ of Fig. 1 the virtualities $k_{1}^{2}, k_{2}^{2} \ll|\hat{t}|$. It means that $q_{t}^{2} \simeq \max \left(k_{1}^{2}, k_{2}^{2}\right)<|\hat{t}|$. Fig. 1 also describes the $\bar{q} q \rightarrow \gamma g$ subprocess but now $k_{2}^{2}=\hat{t}^{\prime}$ plays the role of the hard momentum transfer and strong ordering implies $k_{\bar{q}}^{2}, k_{q}^{2} \ll\left|\hat{t}^{\prime}\right|$. Thus the product $\mathcal{L} d \hat{\sigma} / d \hat{t}$ in (10) should be understood as the sum over the different subprocesses, that is, as $\sum \mathcal{L}_{a b} d \hat{\sigma}_{a b} / d \hat{t}_{a b}$ where $\hat{t}_{g q}=\hat{t}$ and $\hat{t}_{\bar{q} q}=\hat{t}^{\prime}$ in Fig. 11.

The introduction of the delta functions in (10) is simply a technical device to enable the differential cross section to be expressed in terms of $\eta_{\gamma}$ and $p_{t \gamma}^{2}$. Let us study the relevant 


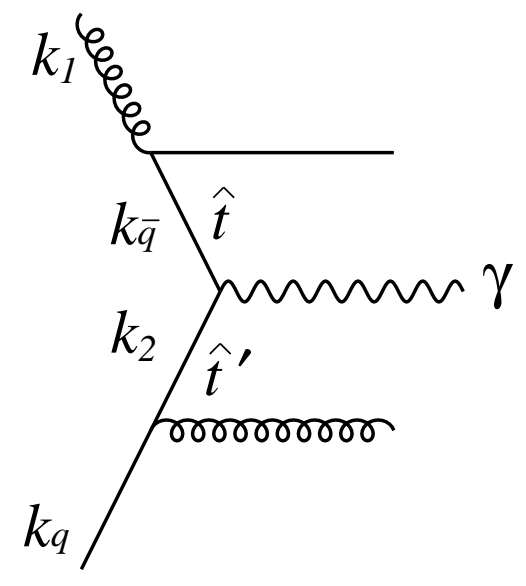

Figure 1: A schematic diagram describing both the subprocesses $g q \rightarrow \gamma q$ and $\bar{q} q \rightarrow \gamma g$, in which the hard momentum transfer squared is either $\hat{t}$ or $\hat{t}^{\prime}$ respectively.

kinematics. If the outgoing $\gamma+$ parton system, of mass $M$ and rapidity $\eta_{M}$, is boosted with the incoming "partonic" transverse momentum $q_{t}$, then the rapidity $\eta_{\gamma}$ and transverse momentum $p_{t \gamma}$ of the photon become

$$
\begin{aligned}
\eta_{\gamma} & =\eta_{M}+\frac{1}{2} \ln \left(\frac{M_{\perp}+q_{s}+M \cos \theta}{M_{\perp}+q_{s}-M \cos \theta}\right), \\
p_{t \gamma}^{2} & =\frac{M^{2}}{4} \sin ^{2} \theta+\frac{\left(q_{t}^{2}+q_{s}^{2}\right)}{4}+\frac{M_{\perp} q_{s}}{2},
\end{aligned}
$$

where

$$
q_{s} \equiv q_{t} \sin \theta \sin \phi, \quad M_{\perp}^{2} \equiv M^{2}+q_{t}^{2}
$$

Equations (12) and (13) specify the missing parts of the delta functions in (10). As the variables $\eta_{M}$ and $M$ have been used to specify the Lorentz boost it is convenient to write the integration over the luminosity in (10) in terms of $d M^{2} d \eta_{M} d q_{t}^{2}$. This is most easily done by noting that

$$
\int \frac{d x_{1}}{x_{1}} \int \frac{d x_{2}}{x_{2}} \delta\left(M^{2}-x_{1} x_{2} s+q_{t}^{2}\right) \delta\left(\eta_{M}-\frac{1}{2} \ln \left(x_{1} / x_{2}\right)\right)=\frac{1}{M_{\perp}^{2}}
$$

We may use the first delta function in (10) to perform the $d \eta_{M}$ integration and the second delta function $\delta\left(p_{t \gamma}^{2}-\ldots\right)$ to perform the $M^{2}$ integration. Then, using $d|\hat{t}|=\frac{1}{2} M^{2} \sin \theta d \theta$, we can rewrite (10) in the form

$$
E_{\gamma} \frac{d \sigma}{d^{3} p_{\gamma}}=\int \mathcal{L}\left(M^{2}, \eta_{M}, q_{t}^{2}\right) \frac{d q_{t}^{2}}{M_{\perp}^{2}} \frac{d \phi}{2 \pi} \frac{d \hat{\sigma}}{d|\hat{t}|} \frac{2 M^{2} d \theta}{\sin \theta+\left(q_{t} / M_{\perp}\right) \sin \phi} \theta\left(\mu^{2}-q_{t}^{2}\right) \theta\left(|\hat{t}|-q_{t}^{2}\right)
$$


with $M_{\perp}$ specified by $\delta\left(p_{t \gamma}^{2}-\ldots\right)$, that is by

$$
M_{\perp}=-q_{t} \frac{\sin \phi}{\sin \theta} \pm \frac{1}{\sin \theta} \sqrt{4 p_{t \gamma}^{2}-q_{t}^{2} \cos ^{2} \theta \cos ^{2} \phi}
$$

Of course we may only use solutions of this latter equation which satisfy $M_{\perp}>q_{t}$.

\section{Effects of partonic $q_{t}$ on the photon spectrum}

It is informative to look at the form of the luminosity function $\mathcal{L}_{g q}$ as a function of the transverse momentum $q_{t}$ of the incoming partonic $(g q)$ system, before we consider the effects of $q_{t}$ on the $p_{t \gamma}$ spectrum of hadroproduced prompt photons. Fig. 2 shows $\mathcal{L}_{g q}$ obtained from (9), for values of $q_{t}$ in the perturbative domain $\left(q_{t}>q_{0}\right)$, at the energies of the UA6 and CDF experiments. We use $\operatorname{MRS}(\mathrm{R} 2)$ partons 15 and take $q_{0}^{2}=1.25 \mathrm{GeV}^{2}$. At the higher Tevatron energy, $\sqrt{s}=1.8 \mathrm{TeV}$, we see that the $q_{t}$ distribution is extensive, whereas at the lower energy, $\sqrt{s}=24.3 \mathrm{GeV}$, the luminosity goes negative for sufficiently large $q_{t}$. At this point we set $\mathcal{L}=0$ for larger values of $q_{t}$. The negative values are an artefact of the double logarithmic approximation on which (9) is based; the derivative $\partial T_{a} / \partial \ln \lambda^{2}$ does not exactly cancel the second (virtual) term in the DGLAP equation (1). The use of the full treatment based on (3) and (7) would not suffer from this defect.

We must, in addition, consider partonic transverse momentum in the domain $q_{t}<q_{0}$. In this region we cannot use perturbative QCD and, moreover, we have no knowledge of the parton distributions at scales $\lambda \lesssim q_{0}$. On the other hand a significant part of the total luminosity comes from the region $q_{t}<q_{0}$ for prompt photon hadroproduction at the lower energies, that is $\sqrt{s} \sim 30 \mathrm{GeV}$. We may quantify this since the integrated luminosity $\mathcal{L}_{0}$ coming from the region $q_{t}<q_{0}$ can be obtained directly from (9)

$$
\mathcal{L}_{0}=a\left(x_{1}, q_{0}^{2}\right) T_{a}\left(q_{0}, \mu\right) b\left(x_{2}, q_{0}^{2}\right) T_{b}\left(q_{0}, \mu\right)
$$

Typical results are given in Table 1 in the relevant energy range. We show the luminosity integrated over the perturbative domain $\left(q_{t}>q_{0}\right)$ together with $\mathcal{L}_{0}$ for given values of $x_{1}=x_{2}$. We have fixed the value of $x_{1}=x_{2}=0.3$ in order to investigate the energy behaviour of the luminosity. For fixed $x_{1}=x_{2}$ and increasing $\sqrt{s}$, the corresponding value of $p_{t \gamma}$ increases and we see that the fraction of the luminosity coming from the non-perturbative region decreases rapidly. At $\sqrt{s}=1800 \mathrm{GeV}$ we also give the luminosity at a more experimentally representative value, $x_{1}=x_{2}=0.03$, where it is much larger.

In order to investigate the effect of the partonic $q_{t}$ on the prompt photon $p_{t \gamma}$ spectrum we need, not just the integrated luminosity $\mathcal{L}_{0}$, but also the $q_{t}$ distribution in the non-perturbative region. We take two extreme possibilities for the distribution in the region $q_{t}<q_{0}$. First, we assume that there is no intrinsic transverse momentum in the non-perturbative region, and set

$$
\mathcal{L}\left(q_{t}<q_{0}\right)=\mathcal{L}_{0} \delta\left(q_{t}^{2}\right)
$$




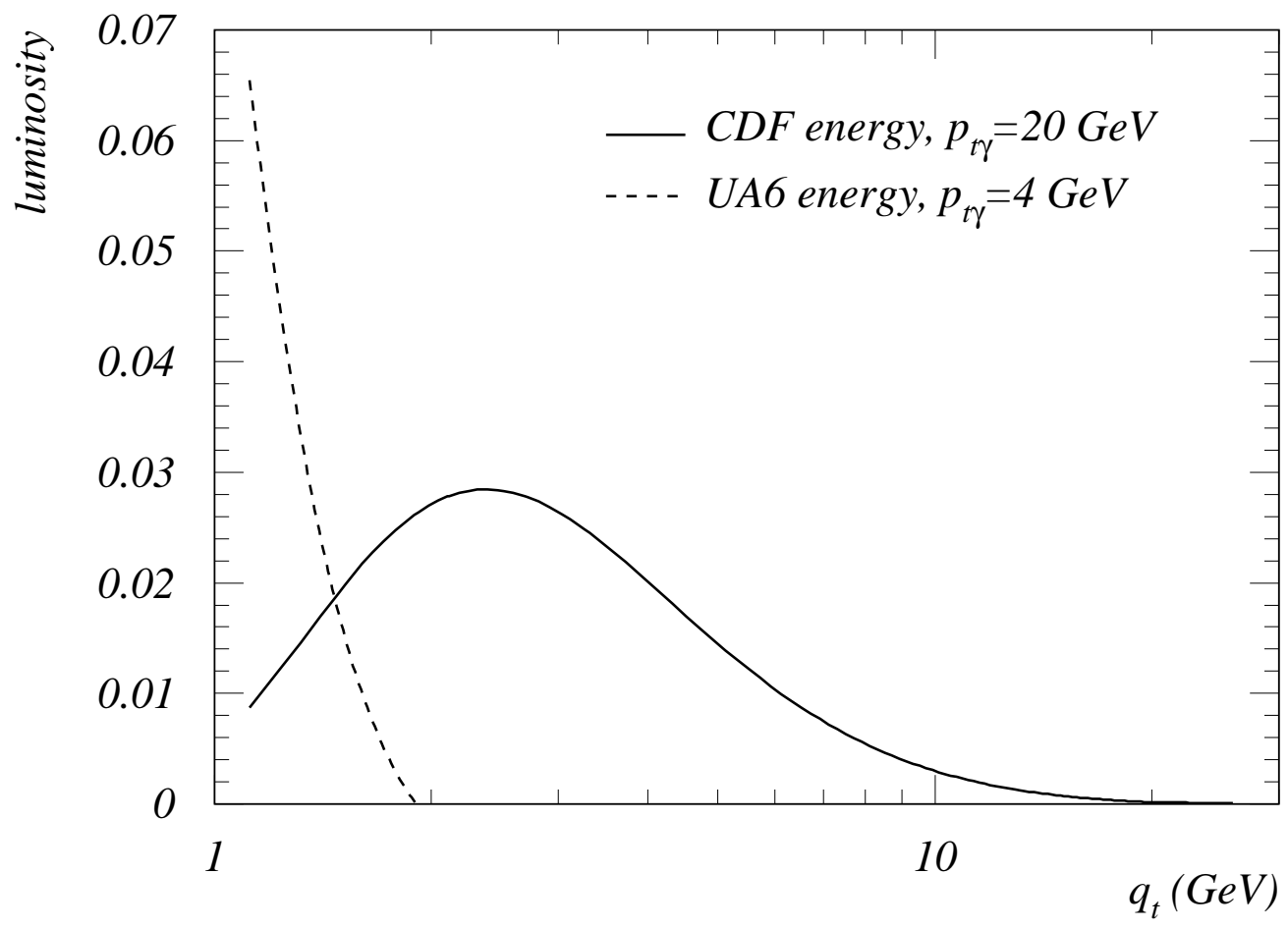

Figure 2: The parton luminosity function $\mathcal{L}_{g q}\left(x_{1}, x_{2}, q_{t}\right)$ of (9) as a function of the incoming partonic $q_{t}$ for $p_{t \gamma}=4 \mathrm{GeV}$ at the UA6 energy of $\sqrt{s}=24.3 \mathrm{GeV}$ and for $p_{t \gamma}=20 \mathrm{GeV}$ at the Tevatron energy of $\sqrt{s}=1800 \mathrm{GeV}$. In each case we take $x_{1}=x_{2}=x_{T}=2 p_{t \gamma} / \sqrt{s}$. The hard scale $\mu$ is taken to be $p_{t \gamma}$. 
Table 1: The integrated luminosity from the perturbative $\left(q_{t}>q_{0}\right)$ and non-perturbative $\left(q_{t}<q_{0}\right)$ domains for typical values of $\sqrt{s}, x_{1}, x_{2}$. We take $q_{0}^{2}=1.25 \mathrm{GeV}^{2}$ and the hard scale $\mu=p_{t \gamma}$.

\begin{tabular}{|c|c|c|c|c|}
\hline$\sqrt{s}(\mathrm{GeV})$ & $x_{1}=x_{2}$ & $\mathcal{L}_{g q}\left(q_{t}>q_{0}\right)$ & $\mathcal{L}_{g q}\left(q_{t}<q_{0}\right)$ & $\begin{array}{l}\text { Fraction from } \\
\qquad q_{t}<q_{0}\end{array}$ \\
\hline 25 & 0.3 & 0.062 & 0.160 & 0.72 \\
\hline 50 & 0.3 & 0.095 & 0.048 & 0.34 \\
\hline 100 & 0.3 & 0.0851 & 0.0103 & 0.11 \\
\hline 500 & 0.3 & 0.0444 & 0.0001 & $2 \times 10^{-3}$ \\
\hline 1800 & 0.3 & 0.0291 & $1.4 \times 10^{-6}$ & $5 \times 10^{-5}$ \\
\hline 1800 & 0.03 & 1.16 & 0.0016 & $1 \times 10^{-3}$ \\
\hline
\end{tabular}

with $\mathcal{L}_{0}$ given by (18). Second, we assume a wide Gaussian distribution of partonic $q_{t}$,

$$
\mathcal{L}\left(q_{t}<q_{0}\right)=\mathcal{L}_{0} \frac{e^{-q_{t}^{2} / q_{0}^{2}}}{q_{0}^{2}\left(1-e^{-1}\right)},
$$

normalized to the region $q_{t}<q_{0}$. From the results shown in Fig. 3 it turns out that the two extreme hypotheses, (19) and (20), for the non-perturbative $q_{t}$ distribution lead to a similar $p_{t \gamma}$ spectrum. Insight into why this result may be anticipated can be obtained from the following simple estimate.

On average the prompt photon gets one half of the $q_{t}$ of the incoming partonic system. Thus the effect of the partonic $q_{t}$ on the hard subprocess cross section is

$$
\begin{aligned}
\frac{d \hat{\sigma}}{d \hat{t}} \sim \frac{1}{p_{t}^{\prime 4}} & =\frac{1}{\left|\boldsymbol{p}_{t \gamma}-\frac{1}{2} \boldsymbol{q}_{t}\right|^{4}} \\
& \rightarrow \frac{1}{p_{t \gamma}^{4}}\left[1+\frac{3\left(\boldsymbol{p}_{t \gamma} \cdot \boldsymbol{q}_{t}\right)^{2}}{p_{t \gamma}^{4}}-\frac{q_{t}^{2}}{2 p_{t \gamma}^{2}}+\ldots\right] \rightarrow \frac{1}{p_{t \gamma}^{4}}\left[1+\frac{q_{t}^{2}}{p_{t \gamma}^{2}}+\ldots\right],
\end{aligned}
$$

after the angular integration. So the relative difference between the $q_{t}<q_{0}$ effects calculated using (19) and (20) is expected to be only about $q_{0}^{2} / 2 p_{t \gamma}^{2}$, since in the non-perturbative domain $\left\langle q_{t}^{2}\right\rangle \lesssim q_{0}^{2}$

At this point one may ask why the traditional phenomenological treatments [6, 7] of the intrinsic partonic $q_{t}$ have found such a large effect. The reason is that the full kinematics were not taken into account. Instead of giving the Lorentz boost to the produced $\gamma+$ parton system, the photon on its own received the total partonic $q_{t}$. To reproduce the same effect with the correct kinematics would require about twice as much partonic $q_{t}$. Moreover strong-ordering of transverse momenta should be imposed which prevents the hard subprocess approaching the $\hat{t} \sim 0$ region. 

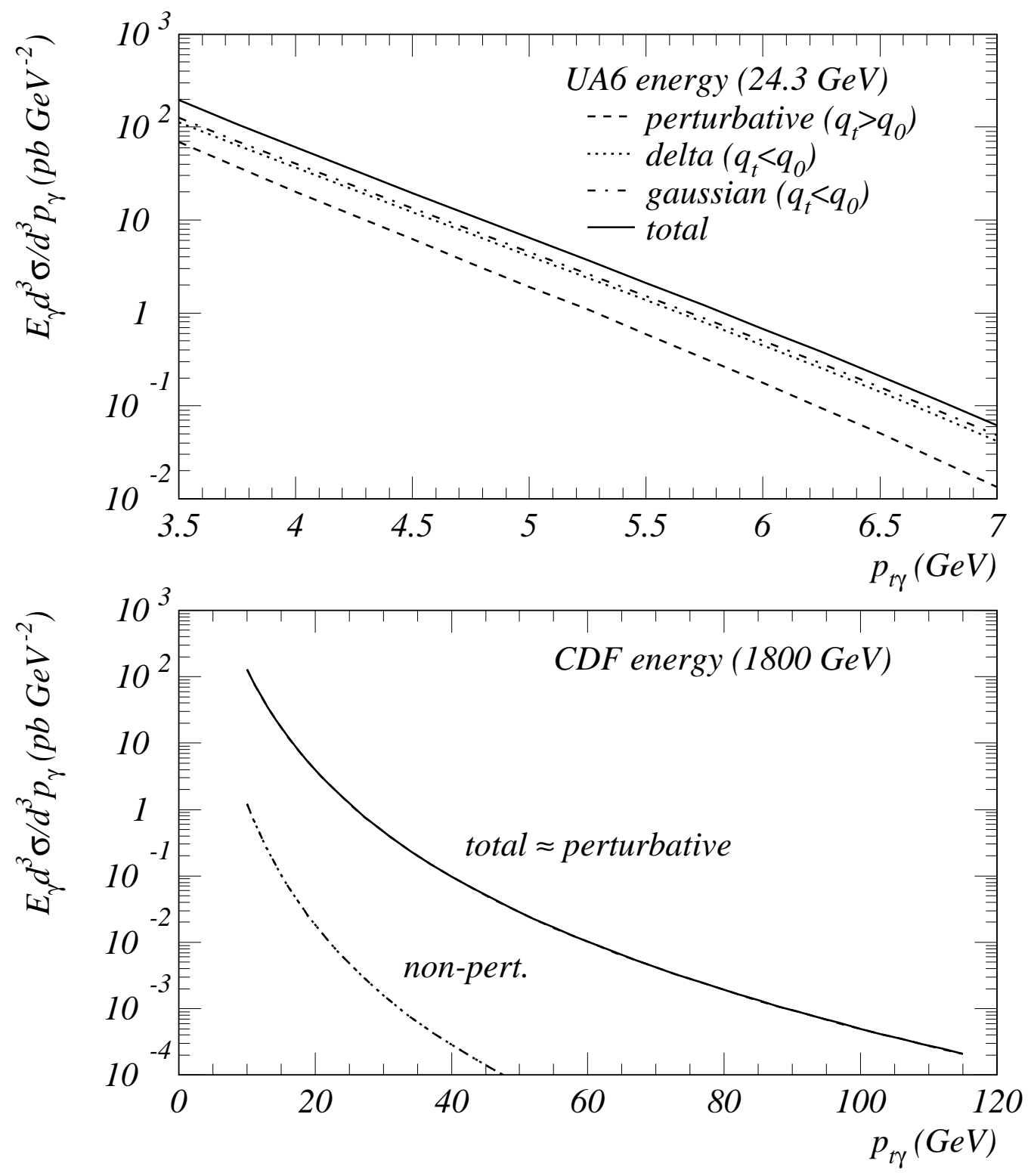

Figure 3: The effect of partonic $q_{t}$ arising from the non-perturbative $\left(q_{t}<q_{0}\right)$, perturbative $\left(q_{t}>q_{0}\right)$, and total contributions to the $p_{t \gamma}$ spectrum of prompt photon production in $p p$ collisions at $\sqrt{s}=24.3 \mathrm{GeV}$ and in $p \bar{p}$ collisions at $1800 \mathrm{GeV}$. Two extreme hypotheses are used for the non-perturbative contribution, but the predictions are very similar (and in fact are indistinguishable on the $\sqrt{s}=1800 \mathrm{GeV}$ plot). We see that the non-perturbative contribution dominates at $\sqrt{s}=24.3 \mathrm{GeV}$, whereas the perturbative dominates at $\sqrt{s}=1800 \mathrm{GeV}$. The hard scale $\mu$ is taken to be $p_{t \gamma}$ and $q_{0}^{2}=1.25 \mathrm{GeV}^{2}$. 
It is informative to discuss the results at the lowest (UA6) and the highest (Tevatron) energies separately. At the lowest energy we see from Fig. 3 that the contribution from the perturbative $\left(q_{t}>q_{0}\right)$ region is relatively small. Thus, even though it depends on the choice of the hard scale $\mu$, the ambiguity is not so important. Moreover we note that the two extreme choices of the non-perturbative $q_{t}$ distribution, (19) and (20), give only a $20 \%$ uncertainty in the $p_{t \gamma}$ spectrum. That is partonic $q_{t}$ smearing has little effect on the $p_{t \gamma}$ spectrum, as may be anticipated from (21). However there are other related points to consider. We see that the formalism requires that the parton densities are sampled at scale $q_{0}$, which enhances the cross section in comparison with the conventional leading order treatment in which the partons are sampled at the hard scale $\mu$. The $q_{t}<q_{0}$ contribution depends on the scale $\mu$ through the survival probabilities $T_{a}$ and $T_{b}$ in $\mathcal{L}_{0}$ of (18). If, for illustration, we take the hard scale $\mu=c p_{t \gamma}$ then, to double log accuracy with fixed $\alpha_{S}$, we have

$$
T_{a} \simeq \exp \left(-\frac{C_{a}}{4 \pi} \alpha_{S}\left(c p_{t \gamma}\right) \ln ^{2}\left(\frac{c p_{t \gamma}}{q_{0}}\right)\right)
$$

Thus an increase in scale, that is a larger $c$, makes $d \sigma / d p_{t \gamma}$ steeper and the cross section smaller. The prediction for the prompt photon spectrum, $d \sigma / d p_{t \gamma}$, at the Tevatron energy is much more certain. Here the contribution for the non-perturbative $q_{t}<q_{0}$ domain is negligible (see Fig. 3), and so the calculation is more under control and is less scale dependent. The difference between using (9) and using an "unsmeared" luminosity

$$
\mathcal{L}_{a b}=\delta\left(q_{t}^{2}\right) a\left(x_{1}, \mu^{2}\right) b\left(x_{2}, \mu^{2}\right)
$$

is not great. The difference is due to the $q_{t}$ smearing itself and also due to the fact that in (9) the partons are sampled at $q_{t}$. This latter effect was not incorporated in the existing treatments of partonic smearing of the $p_{t \gamma}$ distribution.

To demonstrate the sensitivity to the choice of the hard scale, in Fig. 4 we compare the results for $\mu=p_{t \gamma} / 2$ with those for $\mu=p_{t \gamma}$ for three typical energies at which experimental measurements exist. A third choice of the hard scale, $\mu=M / 2$, gives similar results to those obtained with $\mu=p_{t \gamma}$. For comparison the unsmeared cross sections are shown by dashed curves. Very little of the difference between the continuous $\left(q_{t} \neq 0\right)$ and the dashed $\left(q_{t}=0\right)$ curves is directly attributable to the introduction of the partonic transverse momentum $q_{t}$. As mentioned above, the main difference is due to the proper inclusion of the parton densities, which are sampled at scale $q_{t}$ (with $q_{t}>q_{0}$ ) in contrast to the conventional unsmeared result in which they are sampled at the hard scale $\mu$. Of course in a realistic fit to the data we would need to implement the more precise form (3) of the unintegrated parton densities, as well as computing the next-to-leading order expression for the hard subprocess cross sections. However for the largest energy, $\sqrt{s}=1.8 \mathrm{TeV}$, where the $p_{t \gamma}$ is large and leading order is a good approximation, the theoretical uncertainties are seen to be small . In fact the $\mu=p_{t \gamma}$

\footnotetext{
${ }^{4}$ The difference between the predictions for $\mu=p_{t \gamma}$ and $\mu=p_{t \gamma} / 2$ may be considered as an estimate of the size of the next-to- leading order contribution.
} 
prediction gives a reasonable description of the CDF data, particularly at the larger values of $p_{t \gamma}$, see Fig. 5. Figs. 1 and 5 also show that, in common with other analyses? there is a large disagreement between the predictions and the $p_{t \gamma}$ shape of the E706 data.

\section{Conclusions}

This paper should not be regarded as an attempt to better fit data, such as the $p_{t \gamma}$ spectrum of prompt photon hadroproduction, but rather to illustrate the appropriate formalism that should be used to incorporate the transverse momenta of the incoming partons. The crucial point is that, in general, according to the $k_{t}$-factorization prescription [3], cross sections should be calculated in terms of parton distributions, $f_{a}\left(x, k_{t}^{2}, \mu^{2}\right)$, unintegrated over the transverse momentum. Such distributions can be constructed from the conventional parton densities, $a\left(x, \lambda^{2}\right)$, as described in Section 2 .

Prompt photon hadroproduction is an ideal example to illustrate the necessity of using unintegrated parton distributions. For simplicity we chose to work to double logarithm accuracy, rather than the full treatment presented in Section 2. Contrary to the existing phenomenological treatments of the intrinsic transverse momenta of the partons, we find that the $q_{t}$ effects give themselves only a small modification to the $d \sigma / d p_{t \gamma}$ spectrum. Of course there could have been a large effect if $q_{t} \sim p_{t \gamma}$ which would have allowed the singularity of the hard subprocess amplitude to be approached. However the strong ordering of transverse momenta must be incorporated in the formalism, via $\theta\left(|\hat{t}|-q_{t}^{2}\right)$, which prevents this happening. In addition, our study reveals that the $q_{t}$ of the incoming partonic system should be correlated with the scale at which the parton densities are sampled. This fact affects both the shape and the normalization of the predictions, especially at the lower energies.

An interesting result of our study of the parton luminosity is that, at low energy where the contribution from the $q_{t}>q_{0}$ region is small, the factorization procedure is more direct and physically transparent. Recall that we have to introduce a factorization scale to separate the hard subprocess interaction (which is calculated perturbatively) from the universal parton distributions which originate from large distances (that is, from the confinement region). These incoming partons starting at the input scale $q_{0}$ participate in the perturbative evolution which changes the original distributions. However for larger $x$ (that is for the lower energies) we sample the parton densities directly at the low input scale $q_{0}$, see Fig. 2 and (18). From the form of the luminosity we see that essentially no new partons appear in the evolution to larger values of $q_{t}$. The only perturbative effect is the introduction of the survival probability, $T_{a}$, which has a simple physical interpretation and does not alter the kinematics of the partons sampled. This means that the low energy data measure the input distributions directly. On the other hand at the smaller values $]$ of $x$ the perturbative contribution $q_{t}>q_{0}$ dominates. In this case the

\footnotetext{
${ }^{5}$ For example, see the (unsmeared) results in Refs. [4, 5, 6, 7].

${ }^{6}$ For example, UA6 data at $p_{t \gamma}=5 \mathrm{GeV}$.

${ }^{7}$ For example, Tevatron data at $p_{t \gamma}=40 \mathrm{GeV}$.
} 

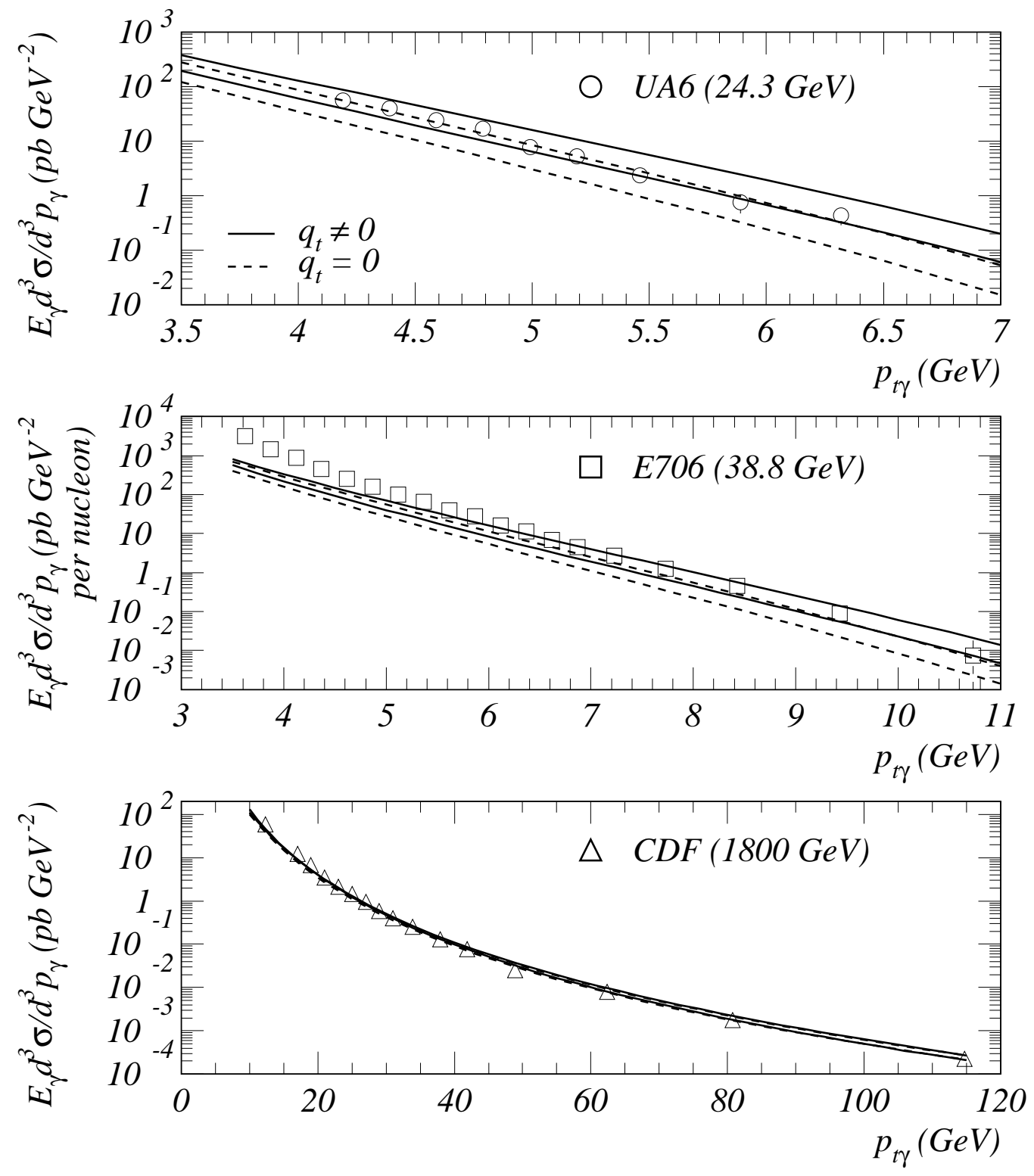

Figure 4: The scale dependence of the predictions for production of prompt photons in $p p$ collisions at $\sqrt{s}=24.3 \mathrm{GeV}, p$ Be collisions at $\sqrt{s}=38.8 \mathrm{GeV}$ and $p \bar{p}$ collisions at $\sqrt{s}=1.8 \mathrm{TeV}$ shown together with UA6 [8], E706 [9] and CDF [10] data respectively. The continuous curves are the predictions with the incoming partonic transverse momentum $q_{t}$ included, whereas the dashed curves correspond to the unsmeared $\left(q_{t}=0\right)$ results in which the integrated partons are sampled at the hard scale $\mu$. In each case the upper curve corresponds to the scale $\mu=p_{t \gamma} / 2$, while the lower corresponds to $\mu=p_{t \gamma}$. 


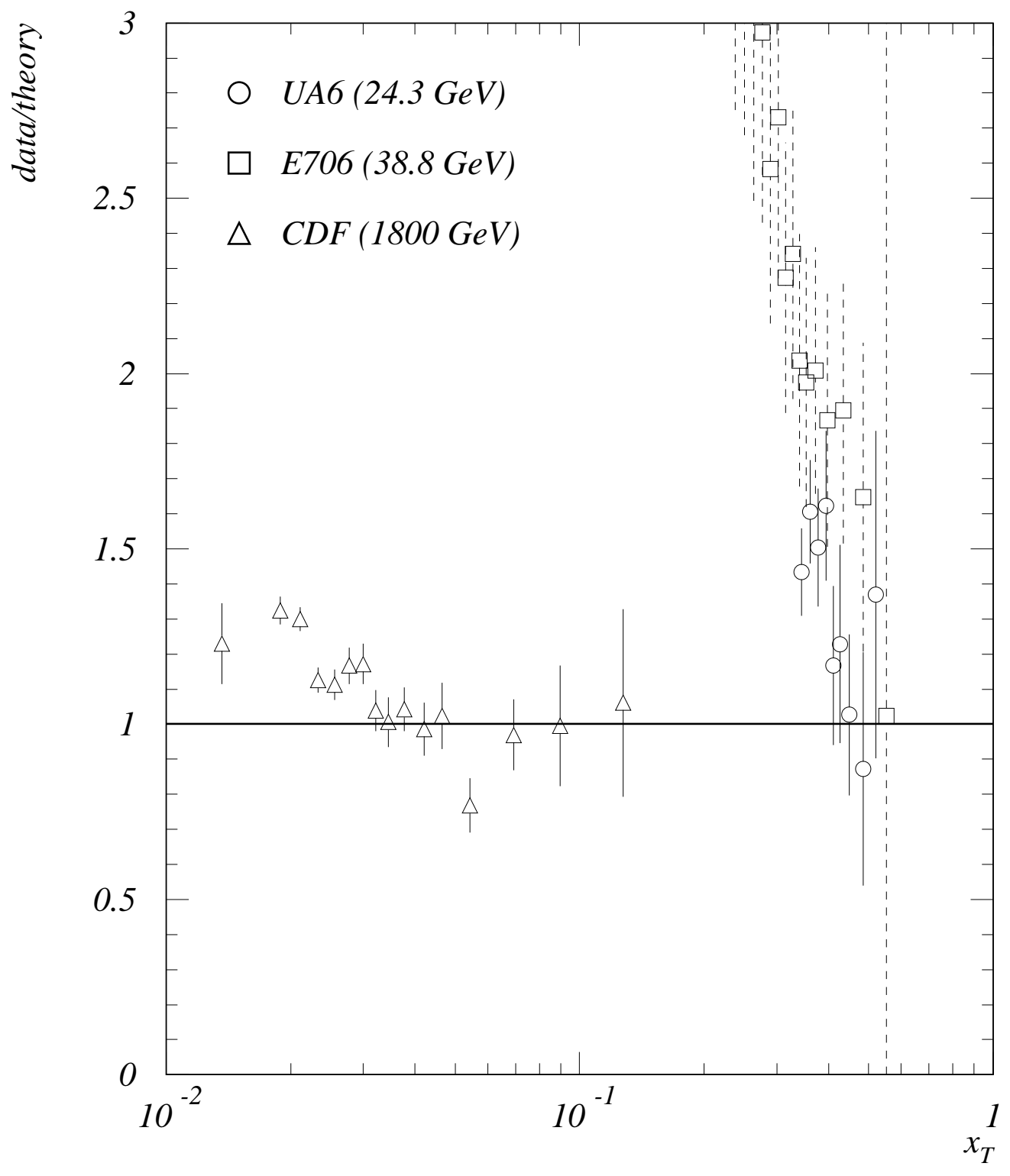

Figure 5: A clearer comparison of the data also plotted in Fig. 1 with our theoretical calculations at the scale $\mu=p_{t \gamma}$; we use $x_{T} \equiv 2 p_{t \gamma} / \sqrt{s}$ as the transverse momentum variable. 
partons evolve from the input scale $q_{0}$ and are finally sampled at the hard factorization scale $\mu \sim p_{t \gamma}$. The evolution of the integrated parton densities in the conventional approach modifies the $x$ behaviour of the distributions assuming that the transverse momenta $k_{t}$ of the partons is small. The formalism that is presented here embodies the modification of the $k_{t}$ distributions of the partons during the evolution, as well as the modification of their $x$ dependence. That is we are able to determine, perturbatively, the $q_{t}=\left|\boldsymbol{k}_{1 t}+\boldsymbol{k}_{2 t}\right|$ distribution of the incoming partonic system, which leaves no room for additional phenomenological $q_{t}$ smearing.

In summary, we have presented the formalism to construct the parton distributions unintegrated over the parton transverse momenta, $f_{a}\left(x, k_{t}^{2}, \mu^{2}\right)$, from the known conventional parton distributions. These distributions should be used to calculate cross sections initiated by hadrons. We applied the formalism to prompt photon hadroproduction in the double logarithm approximation. In this way we are able to quantify the effects of the partonic transverse momentum, $q_{t}$, on the transverse momentum, $p_{t \gamma}$, spectrum of the produced photons. We find that the correct treatment requires that the integrated partons are sampled at the scale $q_{t}$. Clearly this is only meaningful in the perturbative domain $q_{t}>q_{0}$, but we are able to show that the uncertainty due to the contribution from the non-perturbative domain is only of relative size of the order of $q_{0}^{2} / 2 p_{t \gamma}^{2}$.

\section{Acknowledgements}

We thank Dick Roberts for discussions. One of us (MGR) thanks the Royal Society and the Russian Fund for Fundamental Research (98-02-17629) for support. The work was also supported by the UK Particle Physics and Astronomy Research Council and the EU Framework TMR programme, contract FMRX-CT98-0194. 


\section{References}

[1] E.A. Kuraev, L.N. Lipatov and V.S. Fadin, Sov. Phys. JETP 45 (1977) 199;

Ya.Ya. Balitzkij and L.N. Lipatov, Sov. J. Nucl. Phys. 28 (1978) 822;

L.V. Gribov, E.M. Levin and M.G. Ryskin, Phys. Rep. 100 (1983) 1.

[2] Yu.L. Dokshitzer, D.I. Dyakanov and S.I. Troyan, Phys. Rep. 58 (1980) 269.

[3] S. Catani, M. Ciafaloni and F. Hautmann, Phys. Lett. B242 (1990) 97;

Nucl. Phys. B366 (1991) 135;

J.C. Collins and R.K. Ellis, Nucl. Phys. B360 (1991) 3;

G. Marchesini and B.R. Webber, Nucl. Phys. B386 (1992) 215;

S. Catani and F. Hautmann, Nucl. Phys. B427 (1994) 475.

[4] J. Huston et al., Phys. Rev. D51 (1995) 6139.

[5] P. Aurenche, M. Fontannaz, J.Ph. Guillet, B. Kniehl, E. Pilon and M. Werlen, Eur. Phys. J. C9 (1999) 107.

[6] L. Apanasevich et al., Phys. Rev. D59 (1999) 074007.

[7] A.D. Martin, R.G. Roberts, W.J. Stirling and R.S. Thorne, Eur. Phys. J. C4 (1998) 463.

[8] UA6 collaboration, G. Ballocchi et al., Phys. Lett. B436 (1998) 222.

[9] E706 collaboration, L. Apanasevich et al., Phys. Rev. Lett. 81 (1998) 2642.

[10] CDF collaboration, F. Abe et al., Phys. Rev. Lett. 73 (1994) 2662.

[11] S. Catani, M. Mangano, P. Nason, C. Oleari and W. Vogelsang, JHEP 9903:025 (1999).

[12] H.-L. Lai and H.-N. Li, Phys. Rev. D58 (1998) 114020.

[13] CTEQ collaboration: H.-L. Lai et al., hep-ph/9903282.

[14] G. Marchesini and B.R. Webber, Nucl. Phys. B310 (1988) 461.

[15] A.D. Martin, R.G. Roberts and W.J. Stirling, Phys. Lett. B387 (1996) 419. 